\title{
Computational volumetric reconstruction of integral imaging with improved depth resolution considering continuously non-uniform shifting pixels
}

\author{
Byungwoo Cho' ${ }^{1}$, Przemyslaw Kopycki², Manuel Martinez-Corral' ${ }^{2}$, and Myungjin \\ $\mathrm{Cho}^{1, *}$ \\ ${ }^{1}$ Dept. of Electrical, Electronic, and Control Engineering, IITC, Hankyong National University, Anseong-si,456-749, South Korea \\ 2Dept. of Optics, University of Valencia, E-46100, Burjassot, Spain \\ *Corresponding author: mjcho@hknu.ac.kr
}

\begin{abstract}
In this paper, we propose a new computational volumetric reconstruction technique of threedimensional (3D) integral imaging for depth resolution enhancement by using non-uniform and integervalued shifting pixels. In a typical integral imaging system, 3D images can be recorded and visualized using a lenslet array. In previous studies, many computational reconstruction techniques such as computational volumetric reconstruction and pixel of elemental images rearrangement technique (PERT) have been reported. However, a computational volumetric reconstruction technique has low visual quality and depth resolution because low-resolution elemental images and uniformly distributed shifting pixels are used for reconstruction. Although PERT can enhance the visual quality of the 3D images, the size of the reconstructed 3D images is different from the original scene. On the other hand, our proposed method uses non-uniformly distributed shifting pixels for reconstruction instead of uniformly distributed shifting pixels. Therefore, the visual quality and depth resolution may be enhanced. Finally, our experimental results show the improvement of depth resolution and visual quality of the reconstructed 3D images.
\end{abstract}

Keywords: 3D integral imaging, computational volumetric reconstruction, continuously non-uniform shifting pixels, depth resolution 


\section{Introduction}

Three-dimensional (3D) image sensing and visualization of integral imaging has been studied for several applications including unmanned autonomous vehicle system, pattern recognition, 3D entertainments, medical imaging, defense, and so on [1-17]. Integral photography or integral imaging was first proposed by G. Lippmann in 1908 [1]. It has been a popular 3D technique since it can provide full-color, full-parallax, and continuous viewing points without special glasses and coherent light source. In addition, using computational reconstruction [9], 3D information of objects (i.e., 3D point cloud of objects) can be obtained.

A typical integral imaging system is composed of two primary processes: pickup and reconstruction (or display) processes. In the pickup stage, the light rays coming from 3D objects are recorded through a lenslet array on an image sensor such as charge-coupled device (CCD). They are multiple 2D images with different perspectives called as elemental images. In the reconstruction or display stage, a homogeneous lenslet array used in the pickup process is positioned in front of elemental images. Then, the 3D image is formed in free space by backpropagation of light rays for each elemental image.

Recently, many computational volumetric reconstruction techniques are reported; computational volumetric reconstruction [10], 3D profilometry [11], pixel of elemental images rearrangement technique (PERT) [12], PERT with projected empty space (PERTS) [13], and so on [14-17]. In general, computational volumetric reconstruction is used because of its simplicity. In this method, each elemental image is back-projected through the virtual pinhole array on the reconstruction plane. Since typical computational volumetric reconstruction uses the pixilated process and the number of shifting pixels for reconstruction is the fixed integer value and uniformly distributed, the depth resolution of the reconstructed $3 \mathrm{D}$ image is not sufficient. Further, the reconstructed 3D images have poor visual quality when elemental 
images with low resolution are used. To solve these problems, a new reconstruction method is required. Therefore, in this paper, we propose a new computational volumetric reconstruction method with improvement of depth resolution and visual quality for the reconstructed 3D images considering continuously non-uniform shifting pixels when elemental images with low resolution are used. In our proposed method, we use non-uniformly distributed and integervalued shifting pixels for reconstruction. Thus, the depth resolution can be improved compared with the computational volumetric reconstruction method. Also, the visual quality of the reconstructed 3D images can be even enhanced than the conventional reconstruction.

This paper is organized as follows. In Section 2, we briefly present the principle of computational volumetric reconstruction for integral imaging. In Section 3, we explain our proposed method. Then, we show the experimental results to prove that our method can enhance the depth resolution and visual quality compared with the computational volumetric reconstruction method in Section 4. Finally, we conclude with summary in Section 5.

\section{Computational volumetric reconstruction of integral imaging}

In this section, we briefly describe integral imaging system, including pickup and computational volumetric reconstruction processes. In this system, 3D images can be recorded and visualized by the lenslet array under incoherent illumination conditions as shown in Fig. 1. In pickup process, each voxel of the 3D objects is mapped on imaging plane of the lenslets array and recorded by CCD camera. Consequently, each voxel of the 3D objects leads to form all the pixels of elemental images. Since the number of pixels for the image sensor is divided by the number of lenslets, each elemental image has low lateral resolution. Thus, to enhance the lateral resolution of each elemental image, synthetic aperture integral imaging (SAII) [18] as depicted in Fig. 2 can be utilized.

In reconstruction process, there are various computational reconstruction techniques [10-17]. 
In this paper, we consider computational volumetric reconstruction only [10] since it is the most similar with optical reconstruction in integral imaging. The elemental images obtained in pickup process are used in computational volumetric reconstruction as depicted in Fig. 3. In the reconstruction process, the homogeneous lenslet array in the pickup process is used. By back-propagation of the elemental images through the lenslet array in display process, the 3D images are formed in free space. In computational volumetric reconstruction, each elemental image is back-projected through the virtual pinhole array and magnified with magnification ratio $M=z_{d} / f$, where $z_{d}$ is the desired reconstruction depth and $f$ is the focal length of the virtual pinhole. Finally, the reconstructed 3D image can be obtained by averaging all corresponding pixels of elemental images at the reconstruction depth. The procedure of computational volumetric reconstruction is follows [10]:

$$
\begin{gathered}
\Delta x_{s}=\frac{N_{x} \times p_{x} \times f}{c_{x} \times z_{d}}, \quad \Delta y_{s}=\frac{N_{y} \times p_{y} \times f}{c_{y} \times z_{d}} \\
\Delta x_{p}=\left\lceil\Delta x_{s}\right\rfloor, \quad \Delta y_{p}=\left\lceil\Delta y_{s}\right\rfloor \\
I\left(x, y, z_{d}\right)=\frac{1}{O\left(x, y, z_{d}\right)} \sum_{k=0}^{K-1} \sum_{l=0}^{L-1} E_{k l}\left(x+k \Delta x_{p}, y+l \Delta y_{p}\right)
\end{gathered}
$$

where $N_{x}, N_{y}$ are the number of pixels for each elemental image, $p_{x}, p_{y}$ are the pitch between the virtual pinholes, $I\left(x, y, z_{d}\right)$ is the intensity of the reconstructed $3 \mathrm{D}$ image, $O\left(x, y, z_{d}\right)$ is the number of overlaps at the reconstruction plane, $E_{k l}$ is the $k$ th column and $l$ th row elemental image, $c_{x}, c_{y}$ are the size of image sensor, $x, y$ are index of pixels, $\Delta x_{s}, \Delta y_{s}$ are the real-valued shifting pixels of each elemental image for $3 \mathrm{D}$ reconstruction, \lceil\rfloor is the round operator, $\Delta x_{p}, \Delta y_{p}$ are pixilated shifting pixels of $\Delta x_{s}, \Delta y_{s}$, respectively. As shown in Fig. 3, the shifting pixels between elemental images at any arbitrary reconstructed plane $z_{d}$ are all the same and integer in conventional reconstruction method. Therefore, this computational volumetric reconstruction has worse depth resolution and reconstruction error. 


\section{Computational reconstruction of integral imaging with continuously non-uniform shifting pixels}

In integral imaging system, the number of shifting pixels for each elemental image at any arbitrary reconstruction plane $z_{d}$ is not uniform, because the actual value of shifting pixels is real value. However, in the computational volumetric reconstruction method, the accurate depth may not be estimated because the number of shifting pixels for all elemental images is uniformly distributed at any arbitrary reconstruction plane $z_{d}$ (i.e., pixilation). Thus, computational volumetric reconstruction in integral imaging may not obtain the accurate reconstructed 3D image and the depth resolution is inevitably reduced. In addition, the computational volumetric reconstruction has insufficient visual quality when element images with low resolution are used. Therefore, in this paper, we use continuously non-uniform shifting pixels instead of uniform shifting pixels used in computational volumetric reconstruction to improve the depth resolution and visual quality of the reconstructed 3D images. Our technique can be explained as follows:

$$
\begin{gathered}
\Delta x_{s_{k}}=\frac{N_{x} \times p_{x} \times f}{c_{x} \times z_{d}} \times L_{k}, \quad \Delta y_{s_{l}}=\frac{N_{y} \times p_{y} \times f}{c_{y} \times z_{d}} \times L_{l} \\
L_{k}=[0,1, \ldots, K-1], \quad L_{l}=[0,1, \ldots, L-1], \quad \text { for } \begin{array}{r}
k=1,2, \ldots, K \\
l=1,2, \ldots, L
\end{array} \\
\Delta x_{p_{k}}=\left\lceil\Delta x_{s_{k}}\right\rfloor-\left\lceil\Delta x_{s_{k-1}}\right\rfloor, \quad \Delta x_{p_{0}}=0 \\
\Delta y_{p_{l}}=\left\lceil\Delta y_{s_{l}}\right\rfloor-\left\lceil\Delta y_{s_{l-1}}\right\rfloor, \quad \Delta y_{p_{0}}=0
\end{gathered}
$$

where $\Delta x_{s_{k}}, \Delta y_{s_{l}}$ denote the continuously non-uniform shifting pixels for the $k$ th column and the $l$ th row elemental image from the first elemental image (in this paper, the first upper and left elemental image), $L_{k}, L_{l}$ denote the column and row index vectors of virtual pinhole array, 
$\Delta x_{p_{k}}, \Delta y_{p_{l}}$ are pixilated values of the shifting pixels for each elemental image, and $\Delta x_{p 0}, \Delta y_{p 0}$ are the initial shifting pixels for the first elemental image, respectively.

In reconstruction process, the shifting pixel means distance between the virtual pinholes on the reconstruction plane. To explain distance between the virtual pinholes, for example, let us assume that the number of pixel for each elemental image is $300(\mathrm{H}) \times 200(\mathrm{~V}), p_{x}, p_{y}$ are $2 \mathrm{~mm}$, $f$ is $50 \mathrm{~mm}, c_{x}, c_{y}$ are $23.2 \mathrm{~mm}, 15.4 \mathrm{~mm}, K, L$ are 5 and $z_{d}$ is $240 \mathrm{~mm}$, respectively. To compare between conventional computational volumetric reconstruction and our proposed method, we calculate the ratio between shifting pixel values and positions of virtual pinhole array as shown in Fig. 4. Figure 4 illustrates a comparison of the positions of virtual pinhole array for ideal computational volumetric reconstruction, conventional computational volumetric reconstruction, and our proposed method. The black circle indicates the virtual pinholes of the ideal computational volumetric reconstruction, the blue square indicates the virtual pinholes of the conventional computational volumetric reconstruction, and the red star indicates the virtual pinholes of our proposed method. In conventional computational volumetric reconstruction, all of the shifting pixels $\Delta x_{p}$ and $\Delta y_{p}$ for elemental images are the same by Eqs. (1)-(3) at the reconstruction depth $z_{d}$. Thus, as shown in Fig. 4, position difference of virtual pinholes between ideal computational volumetric reconstruction and conventional computational volumetric reconstruction is very large. Therefore, the reconstructed 3D image by conventional computational volumetric reconstruction has low depth resolution and high reconstruction error. On the other hand, in our proposed method, the shifting pixels $\Delta x_{p_{k}}$ and $\Delta y_{p_{l}}$ for $k$ th the column and the $l$ th row elemental image are continuously non-uniform by Eqs. (4)-(6) at the reconstruction depth $z_{d}$. Accordingly, as shown in Fig. 4, position difference of virtual pinholes between ideal computational volumetric reconstruction and our proposed method is relatively small. It is indicated that virtual pinhole positions in our proposed method are closer to the 
ideal computational volumetric reconstruction than conventional computational volumetric reconstruction. Therefore, our proposed method can obtain the enhanced depth resolution and the visual quality of the reconstructed $3 \mathrm{D}$ image.

\section{Experimental results}

To evaluate the performance of our proposed method, we first implement computer simulation by Autodesk 3DS Max with object, an ISO 12233 test chart [19]. In this simulation, $9(\mathrm{H}) \times 9(\mathrm{~V})$ camera array are set up with identical focal length, $50 \mathrm{~mm}$. The pitch between two neighboring cameras is $2 \mathrm{~mm}$ in both $x$ and $y$ directions. The distance of the ISO 12233 test chart from the camera is $150 \mathrm{~mm}$. The sensor size is $36 \mathrm{~mm}(\mathrm{H}) \times 36 \mathrm{~mm}(\mathrm{~V})$. Figure 5 shows the recorded elemental images.

Figure 6 shows the reconstructed 3D images by conventional computational volumetric reconstruction and our proposed method. In conventional volumetric reconstruction, the reconstructed 3D image has very low visual quality as shown in Fig. 6(b). It indicates that the reconstructed $3 \mathrm{D}$ image has high reconstruction error. On the other hand, in our proposed method, the reconstructed 3D image as shown in Fig. 6(c) has a better visual quality than the reconstructed 3D image of conventional volumetric reconstruction. To show the visual quality enhancement of our proposed method, we enlarged some parts of ISO 12233 as shown in Fig. 6(d). Thus, it can be seen that our proposed method can enhance the visual quality.

To further verify the depth resolution and visual quality enhancement of our proposed method, we implement the optical experiments. In the experiment, three toy cars are used as 3D scene illuminating the incoherent light source as shown in Fig. 7. The distances of three objects from the camera are approximately $225 \mathrm{~mm}, 280 \mathrm{~mm}$, and $340 \mathrm{~mm}$, respectively. The "3D" character of the first object is located at approximately $240 \mathrm{~mm}$ and the " 287 " and "FIRE" characters of the second object are located at approximately $286 \mathrm{~mm}$ and $300 \mathrm{~mm}$, respectively. And lastly, 
the "POLICE" character of the third object is located at approximately $363 \mathrm{~mm}$. Elemental images are captured with SAII. The focal length of the image sensor is $50 \mathrm{~mm}$, and the sensor size is $23.2 \mathrm{~mm}(\mathrm{H}) \times 15.4 \mathrm{~mm}(\mathrm{~V})$. The pitch between cameras is $2 \mathrm{~mm}$ in both $x$ and $y$ directions. Each elemental image has $3008(\mathrm{H}) \times 2000(\mathrm{~V})$ pixels and the total number of elemental images is $16(\mathrm{H}) \times 16(\mathrm{~V})$. However, in this paper, we use elemental images with $300(\mathrm{H}) \times 200(\mathrm{~V})$ pixels because we need to show the improvement of both depth resolution and visual quality when low-resolution elemental images are used.

Figure 8 shows the reconstructed 3D images by conventional computational volumetric reconstruction and our method. In the ideal computational volumetric reconstruction, focused parts at the actual reconstruction depth can be obtained. However, in conventional computational volumetric reconstruction with low-resolution elemental images, the reconstructed 3D images may not be generated at the correct reconstruction depth $z_{d}$ as shown in Fig. 8(a). It means that the depth resolution is low and discontinuous. On the other hand, in our proposed method, the reconstructed 3D images can be obtained at the correct reconstruction depth $z_{d}$ as shown in Fig. 8(b). Therefore, it can be seen that our method can improve the depth resolution. In addition, to compare the accuracy of the reconstructed $3 \mathrm{D}$ images between our proposed method and conventional computational volumetric reconstruction, we calculate the cross-correlations using phase-only filter (POF) [20]. The POF is defined as:

$$
\begin{gathered}
H_{\phi}(\alpha, \beta)=\exp \left[-i \phi_{R}(\alpha, \beta)\right] \\
g(x, y)=F^{-1}\left\{K(\alpha, \beta) H_{\phi}(\alpha, \beta)\right\}
\end{gathered}
$$

where $H_{\Phi}(\alpha, \beta)$ is the complex conjugate of the phase in the spatial frequency spectrum of the reference images as shown in Fig. $8(\mathrm{c})$ and $K(\alpha, \beta)$ is the Fourier transform of the reconstructed 3D images as shown in Fig. 8(a) and Fig. 8(b) (i.e., red box). $g(x, y)$ is the cross-correlation between the reference images and the reconstructed 3D images. The peak sidelobe ratio (PSR) 
can be calculated to evaluate the reconstruction accuracy. The higher the value of PSR, the more reconstruction accuracy can be obtained. The PSR is defined as:

$$
P S R=\frac{\max [g(x, y)]-\bar{g}(x, y)}{\sqrt{\operatorname{var}[g(x, y)]}}
$$

where $\bar{g}(x, y)$ is the average value of cross-correlation between the reference image and the reconstructed 3D image, $\sqrt{\operatorname{var}[g(x, y)]}$ is the standard deviation of cross-correlation. Figure 9 shows the reconstruction accuracy for both conventional computational volumetric reconstruction and our proposed method.

In Fig. 9, the higher similarity between the reference image and the target of the reconstructed 3D image, the higher PSR value can be obtained. In conventional computational volumetric reconstruction, the PSR values are very low and do not change continuously. It is noticed that the reconstructed 3D images by conventional computational volumetric reconstruction have very low accuracy. On the other hand, in our proposed method, the PSR values are very high at actual depth and vary continuously throughout the reconstruction planes. It means that the reconstructed 3D images by our method have much higher reconstruction accuracy than conventional computational volumetric reconstruction.

Figure 10 shows the measured depths of the reconstructed 3D images by both conventional computational volumetric reconstruction and our proposed method. The red dotted line indicates the conventional computational volumetric reconstruction and the blue line indicates our proposed method. The measured depths by conventional computational volumetric reconstruction change discontinuously. On the other hand, the measured depths by our proposed method change continuously. It means that our proposed method can enhance the depth resolution of the reconstructed 3D images. Therefore, our proposed method can be used in many applications that require precise depth resolution such as microscopy system [21-22], 
semiconductor inspection equipment, and so on.

\section{Conclusion}

In this paper, we have proposed a new computational reconstruction technique of integral imaging with non-uniformly distributed and integer-valued shifting pixels. Conventional computational volumetric reconstruction technique has high reconstruction error and low depth resolution. On the other hand, our proposed method can solve these problems using nonuniformly distributed and integer-valued shifting pixels. Since continuous shifting pixel difference among elemental images in our proposed method makes the reconstructed 3D image to be changed, the depth resolution can be improved. However, in our method, we do not consider the sub-pixel overlapping between adjacent elemental images on reconstruction plane because integer-valued shifting pixels are used. We believe that our proposed method with subpixel overlapping can obtain better visual quality and depth resolution of the reconstructed 3D images. Therefore, in future work, we will investigate a computational volumetric reconstruction method considering this issue.

\section{Acknowledgement}

This research was supported by Basic Science Research Program through the National Research Foundation of Korea(NRF) funded by the Ministry of Education(NRF2017R1D1A1B03030343).

\section{References}

1. G. Lippmann, "La photographie integrale," C. R. Acad. Sci. 146, 446-451 (1908).

2. T. Okoshi, “Three-dimensional Displays,” Proc. IEEE 68, 548-564 (1980).

3. A. Stern and B. Javidi, "Three-dimensional image sensing, visualization, and processing using integral imaging," Proc. IEEE 94, 591-607 (2006). 
4. B. Javidi and S.-H. Hong, "Three-dimensional holographic image sensing and integral imaging display," J. of Display Technol. 1, 341-345 (2005).

5. M. Martinez-Corral, B. Javidi, R. Martinez-Cuenca, and G. Saavedra, "Integral imaging with improved depth of field by use of amplitude modulated microlens array," Appl. Opt. 43, 5806-5813 (2004).

6. J. Arai, F. Okano, H. Hoshino, and I. Yuyama, "Gradient-index lens-array method based on real-time integral photography for three-dimensional images," Appl. Opt. 37, 20342045 (1998).

7. M. Cho, M. Daneshpanah, I. Moon, and B. Javidi, "Three-dimensional optical sensing and visualization using integral imaging," Proc. IEEE 99, 556-575 (2011).

8. C. B. Burckhardt, "Optimum parameter and resolution limitation of integral photography," J. Opt. Soc. Amer. 58, 71-76 (1968).

9. H. Arimoto and B. javidi, "integral three-dimensional imaging with digital reconstruction," Opt. Lett. 26, 157-159 (2001).

10. S.-H. Hong, J.-S. Jang and B. Javidi, "Three-dimensional volumetric object reconstruction using computational integral imaging," Opt. Express 12, 483-491 (2004).

11. M. Daneshpanah and B. Javidi, "Profilometry and optical slicing by passive threedimensional imaging," Opt. Lett. 34, 1105-1107 (2009).

12. M. Cho and B. Javidi, "Computational reconstruction of three-dimensional integral imaging by rearrangement of elemental image pixels," J. of Display Technol. 5, 61-65 (2009).

13. K. Inoue, M. C. Lee, B. Javidi, and M. Cho, "Improved 3D integral imaging reconstruction with elemental image pixel rearrangement," J. of Optics. 20, 025703 (2018).

14. D. Shin, C. -W. Tan, B. -G. Lee, J. -J. Lee, and E. -S. Kim, "Resolution-enhanced threedimensional image reconstruction by use of smart pixel mapping in computational integral 
imaging," Appl. Opt. 47, 6656-6665 (2008).

15. J. Y. Jang and M. Cho, "Fast computational integral imaging reconstruction by combined use of spatial filtering and rearrangement of elemental image pixels," Opt. Laser. Eng. 75, 57-62 (2015).

16. Y. Piao, M. Qu, M. Zhang, and M. Cho, "Three-dimensional integral imaging display system via off-axially distributed image sensing," Opt. Laser. Eng. 85, 18-23 (2016).

17. M. C. Lee, J. Han, and M. Cho, “3D visualization technique for occluded objects in integral imaging using modified smart pixel mapping," J. Inf. Commun. Convergence Eng. 15, 256261 (2017).

18. J.-S. Jang and B. Javidi, "Three-dimensional synthetic aperture integral imaging," Opt. Lett. 27, 1144-1146 (2002).

19. Westin, S. H., "ISO12233 Test Chart,” http://stephen-westin.com/misc/res-chart.html (19 March 2018).

20. J. L. Horner and P. D. Gianino, "Phase-only matched filtering," Appl. Opt. 23, 812-863 (1984)

21. J.-S. Jang and B. Javidi, "Three-dimensional integral imaging of micro-objects," Opt. Lett. 29, 1230-1232 (2004).

22. B. Javidi, I. Moon, and S. Yeom, "Three-dimensional identification of biological microorganism using integral imaging," Opt. Express 14, 12096-12108 (2006).

\section{Figure Captions}

Fig. 1. Concept of conventional integral imaging system.

Fig. 2. Synthetic aperture integral imaging (SAII).

Fig. 3. Computational volumetric reconstruction process.

Fig. 4. Comparison of the positions of virtual pinhole array for ideal computational volumetric reconstruction, conventional computational volumetric reconstruction, and 
our proposed method.

Fig. 5. Elemental images generated by computer graphic software such as Autodesk 3DS Max.

Fig. 6. Simulation results. (a) Center-view elemental image, (b) conventional computational volumetric reconstruction, (c) our proposed method, and (d) enlarged parts of ISO 12233 for comparison of visual quality. Red, green, and blue boxes mean center-view elemental image, conventional computational volumetric reconstruction, and our proposed method, respectively.

Fig. 7. Experimental setup of integral imaging system.

Fig. 8. Reconstruction results. (a) Conventional computational volumetric reconstruction, (b) our proposed method, and (c) center-view elemental image as reference.

Fig. 9. Peak to sidelobe ratio (PSR) via various reconstruction depths by using conventional computational volumetric reconstruction and our proposed method.

Fig. 10. Measured depths by conventional computational volumetric reconstruction and our proposed method. 


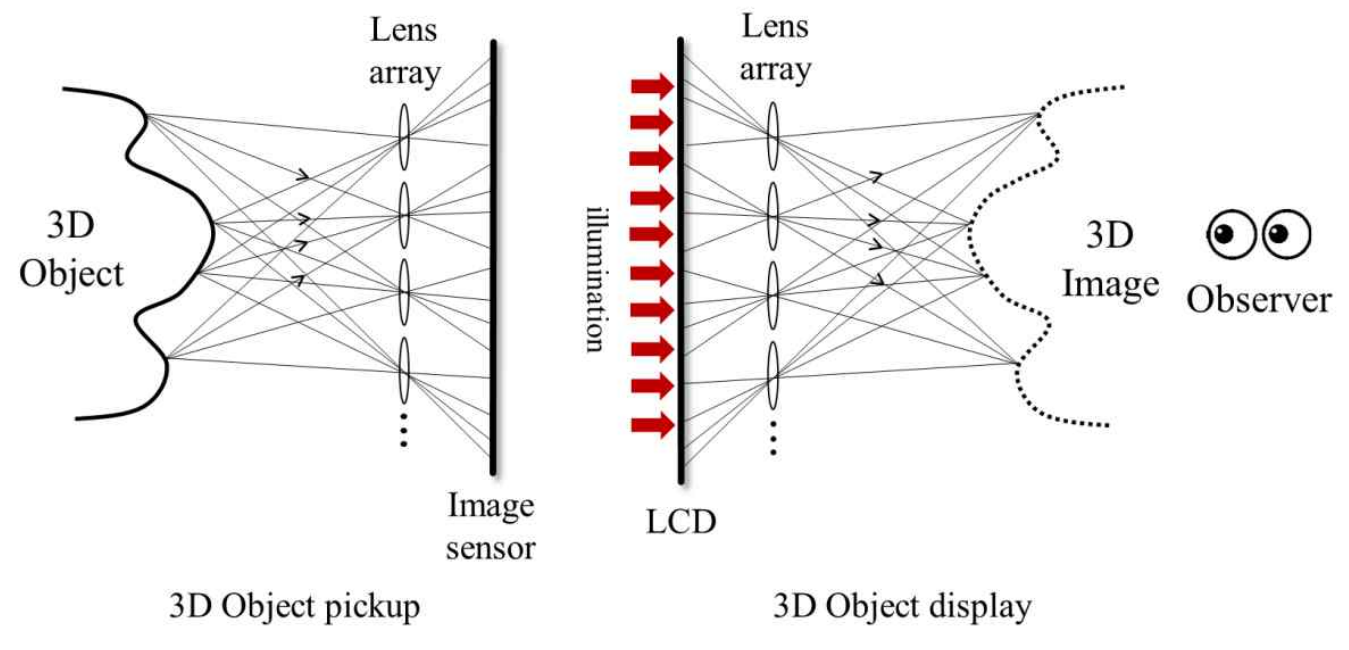

Figure 1. 


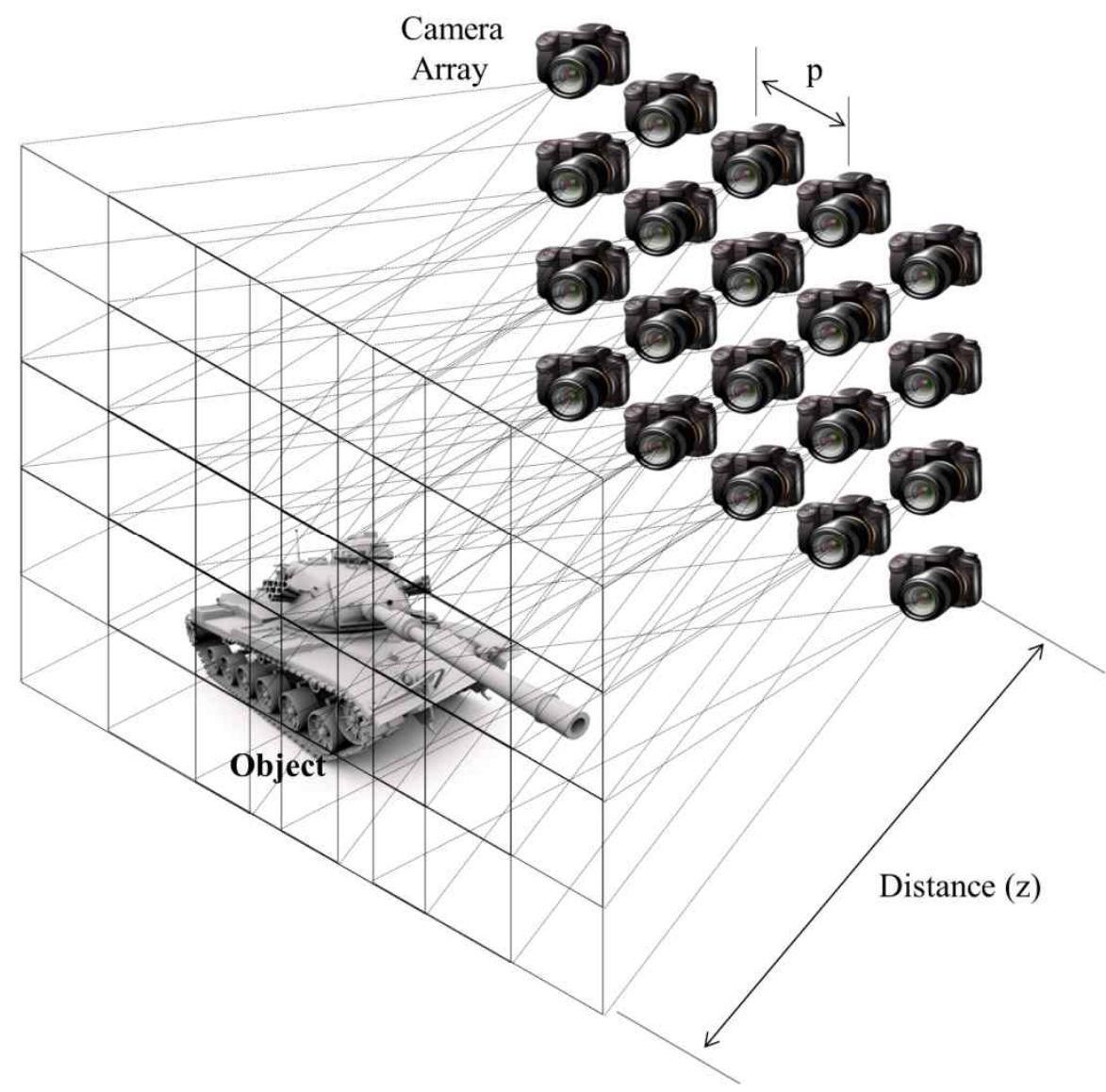

Figure 2. 


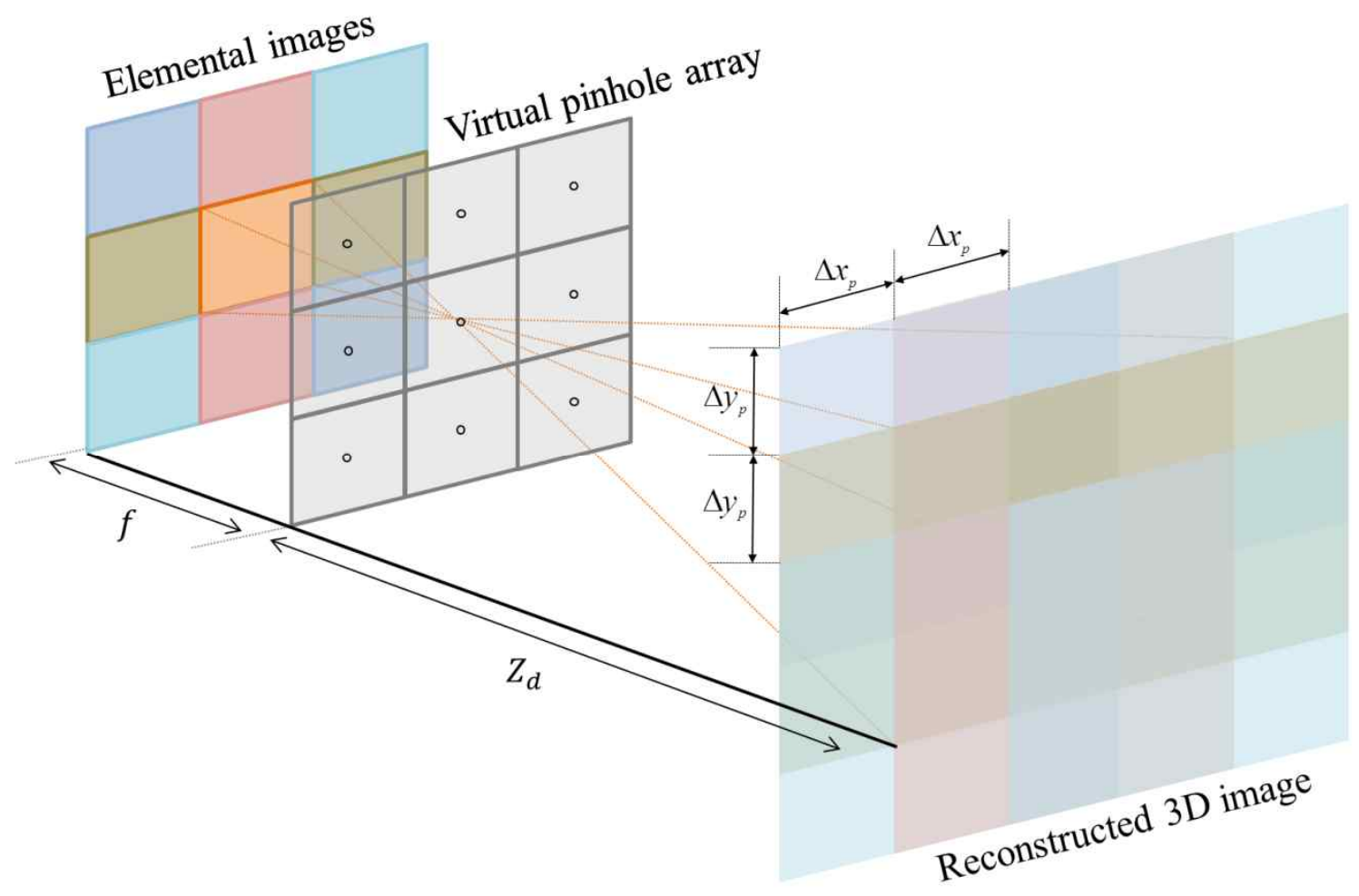

Figure 3. 


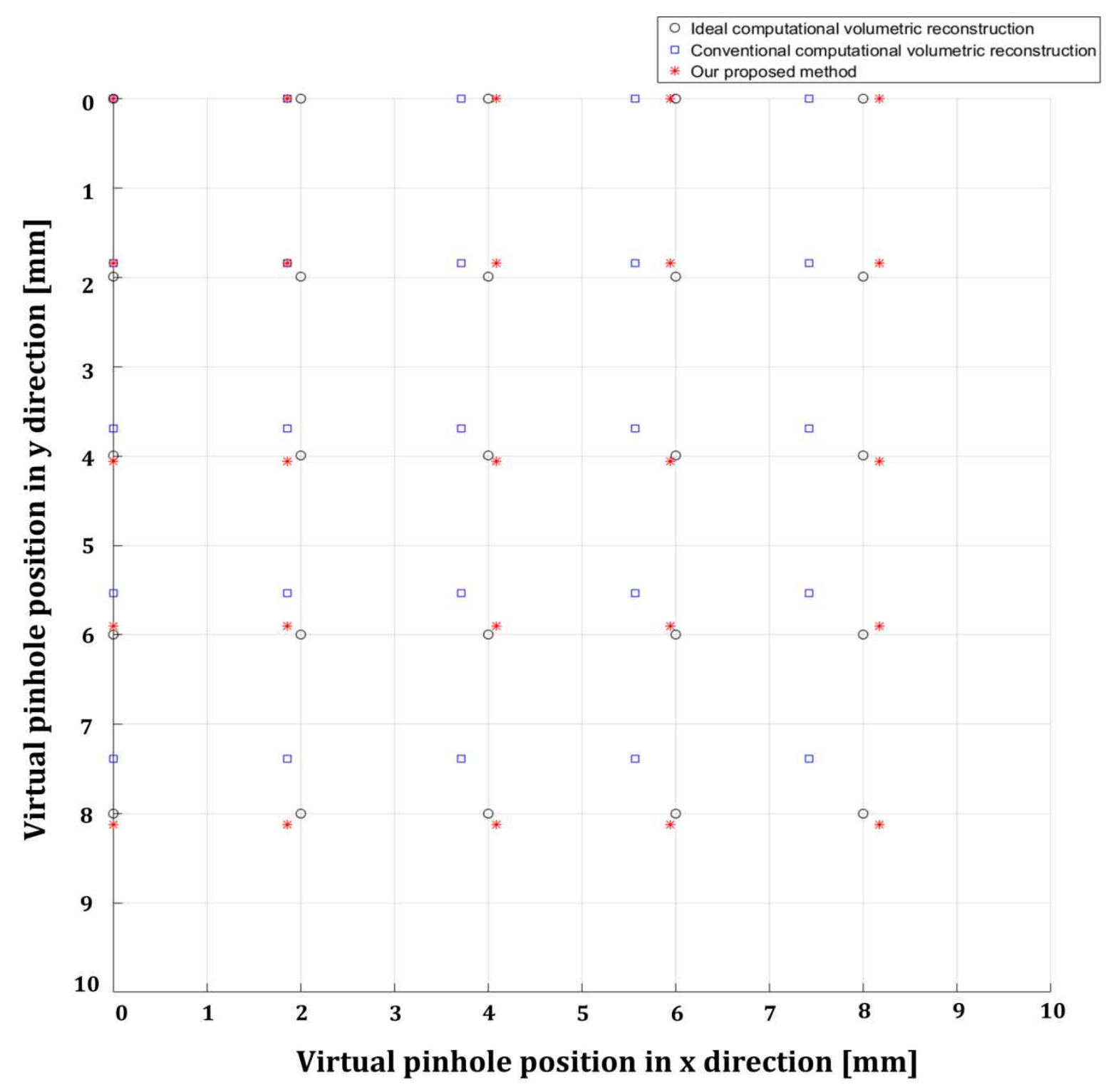

Figure 4. 

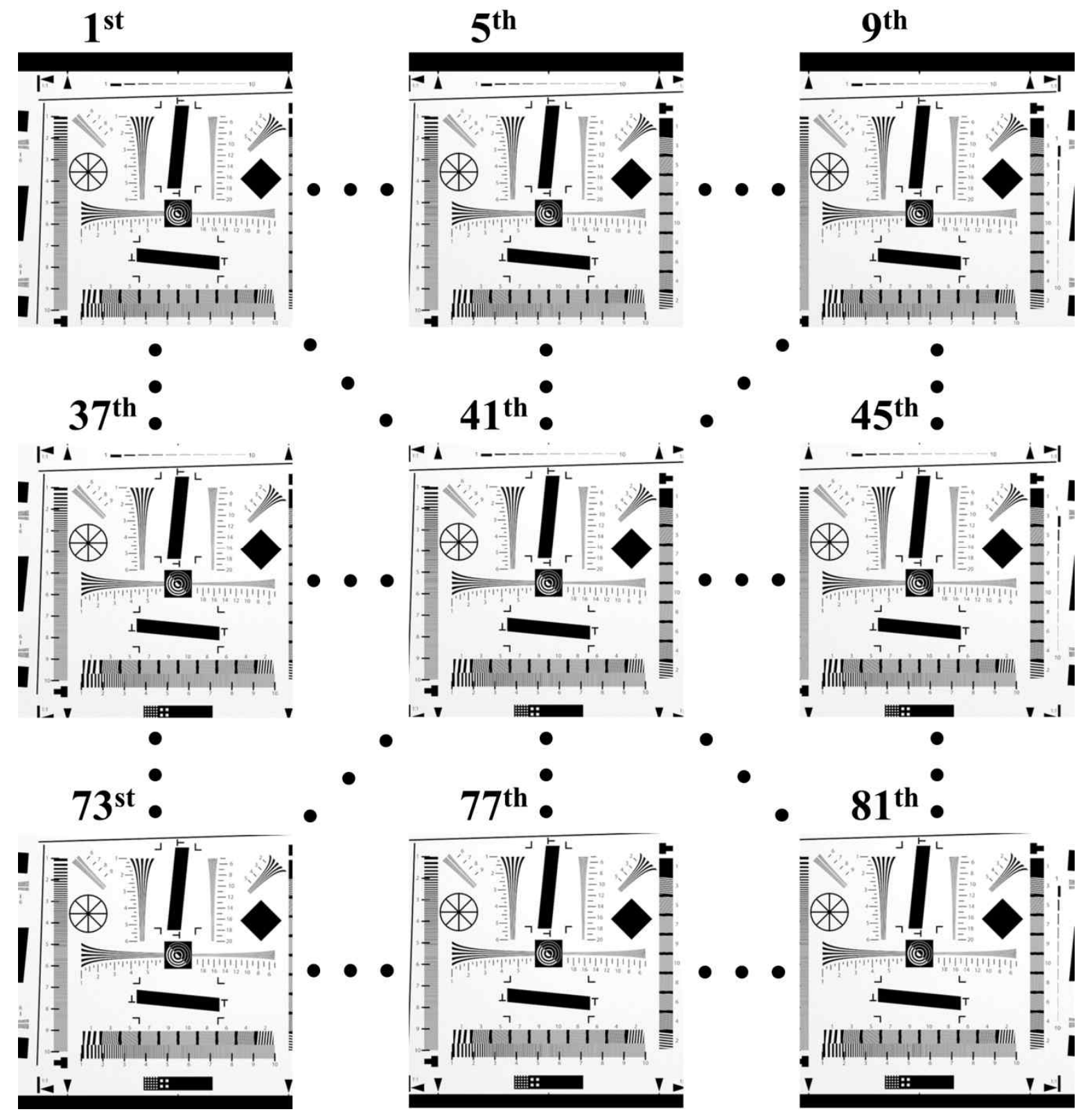

Figure 5. 


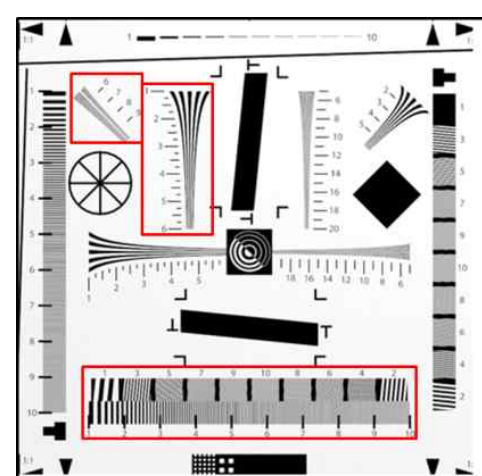

(a)

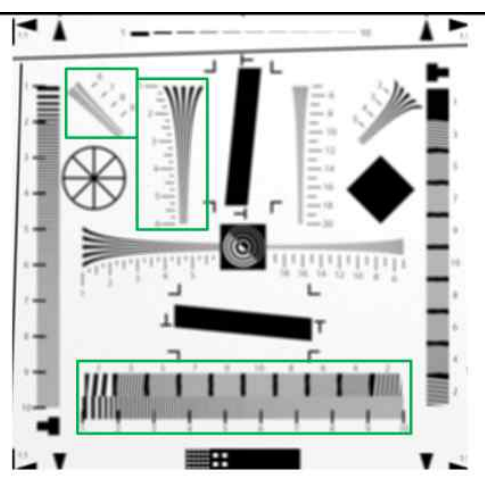

(b)

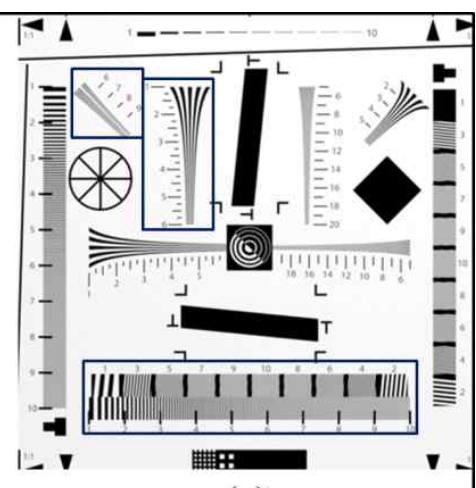

(c)
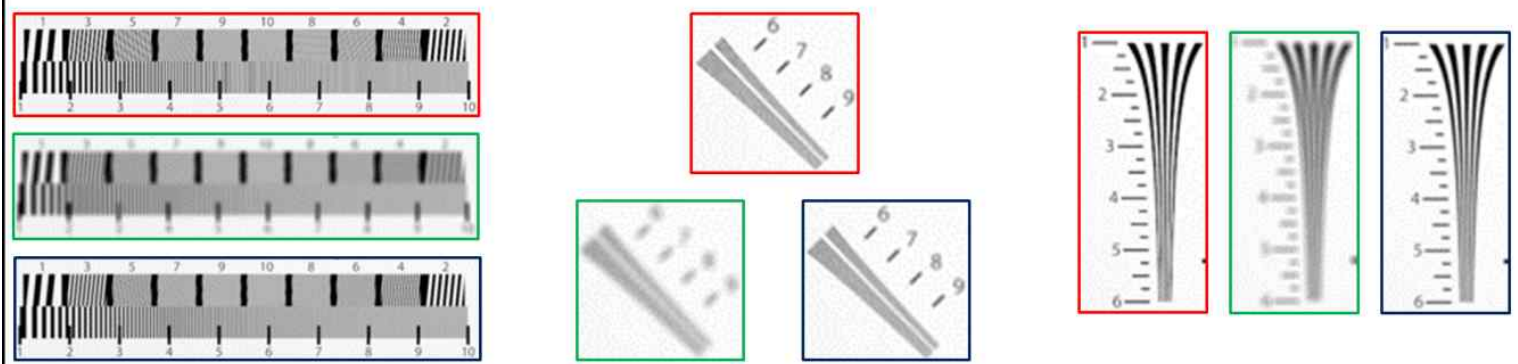

(d)

Figure 6. 


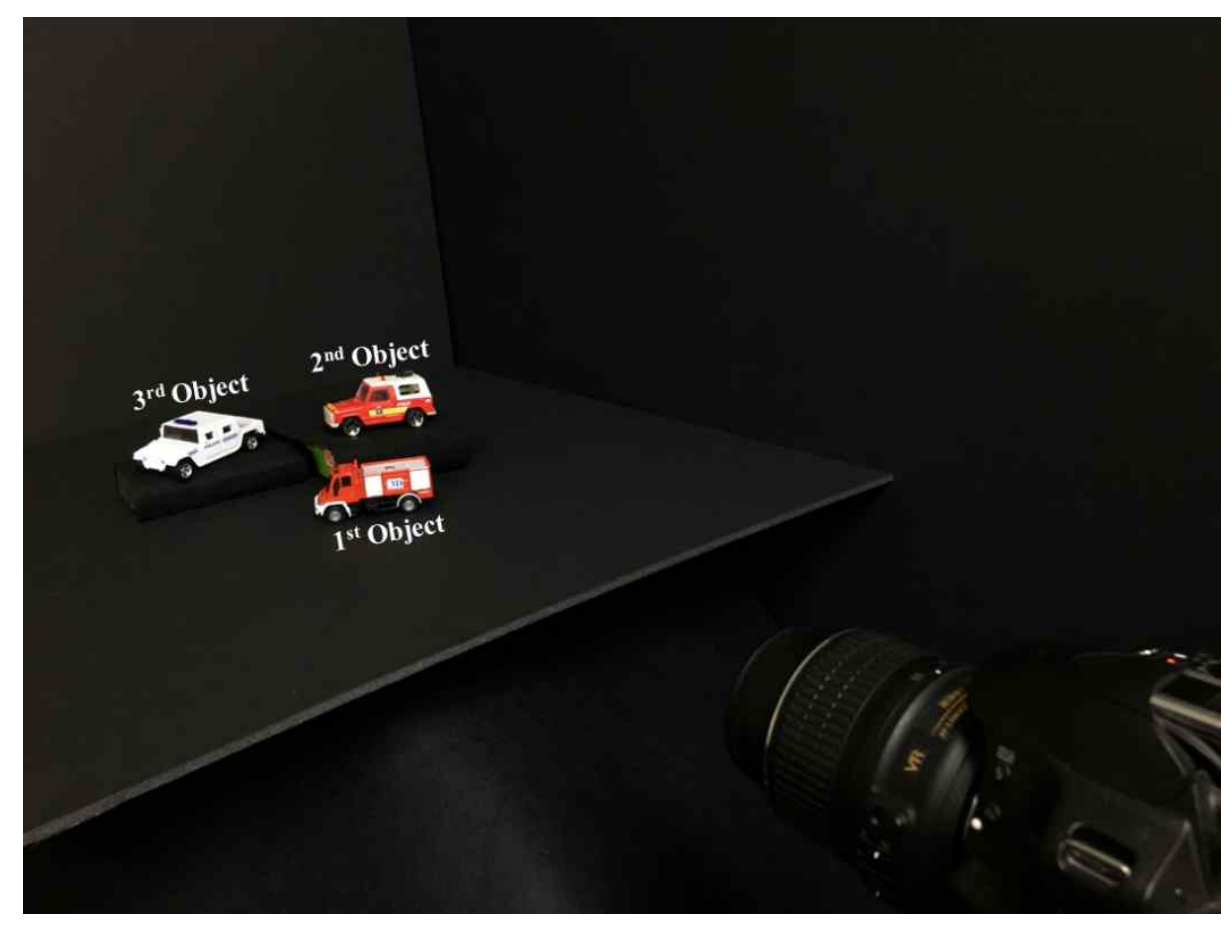

Figure 7. 


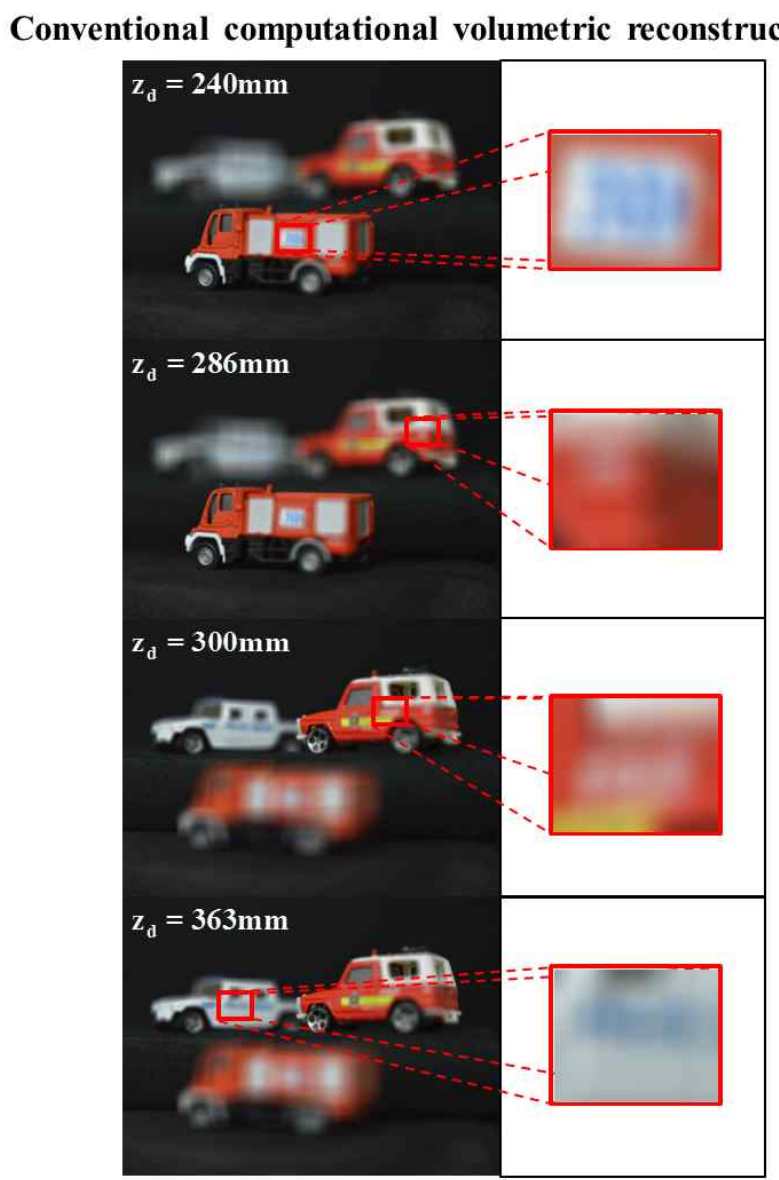

(a)

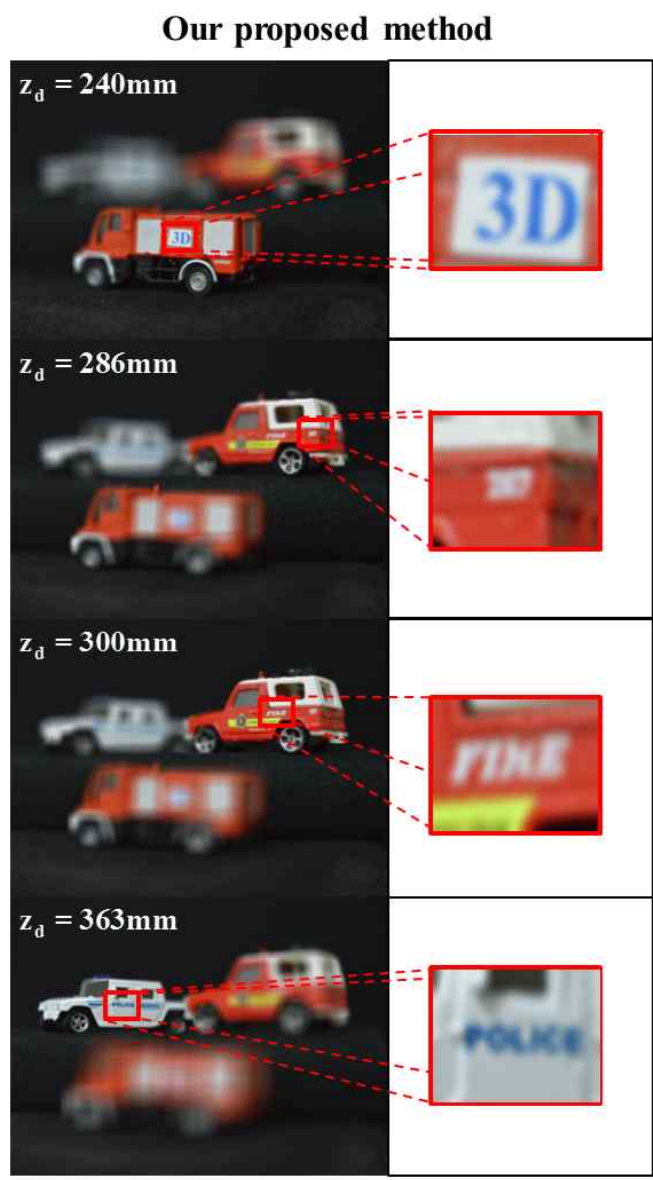

(b)

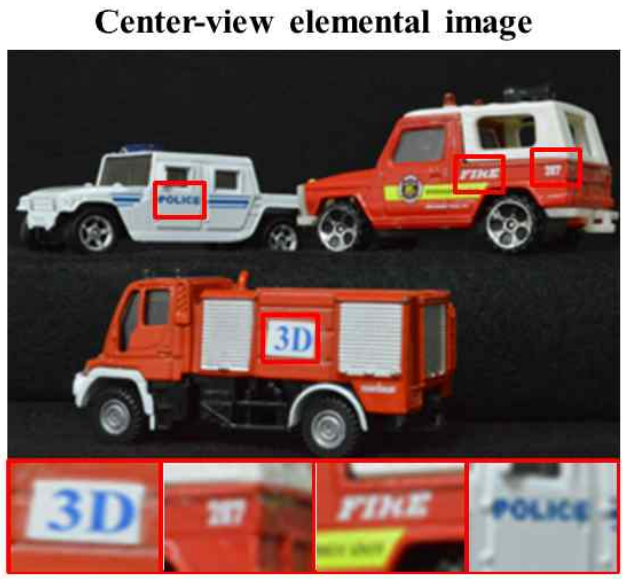

(c)

Figure 8. 


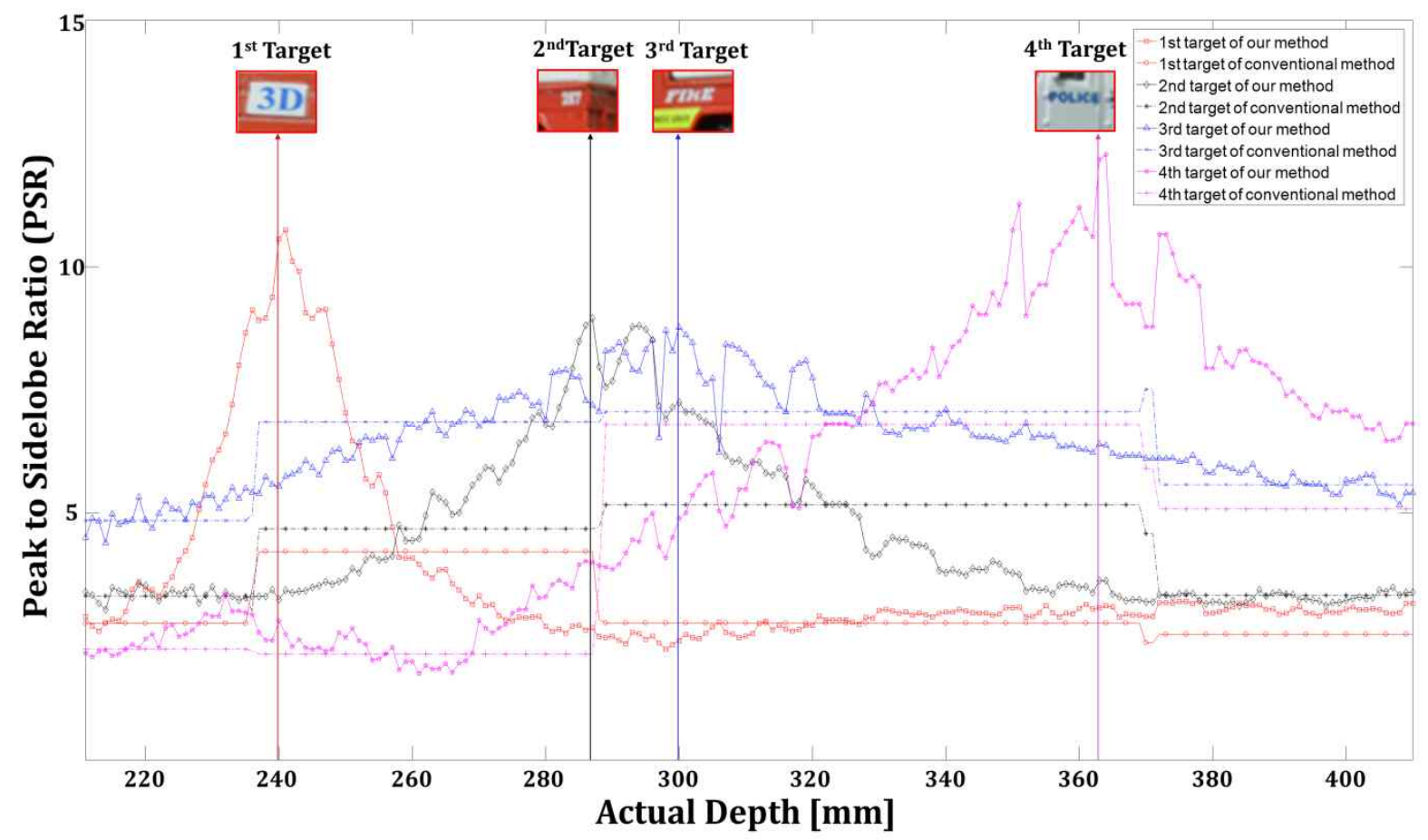

Figure 9. 


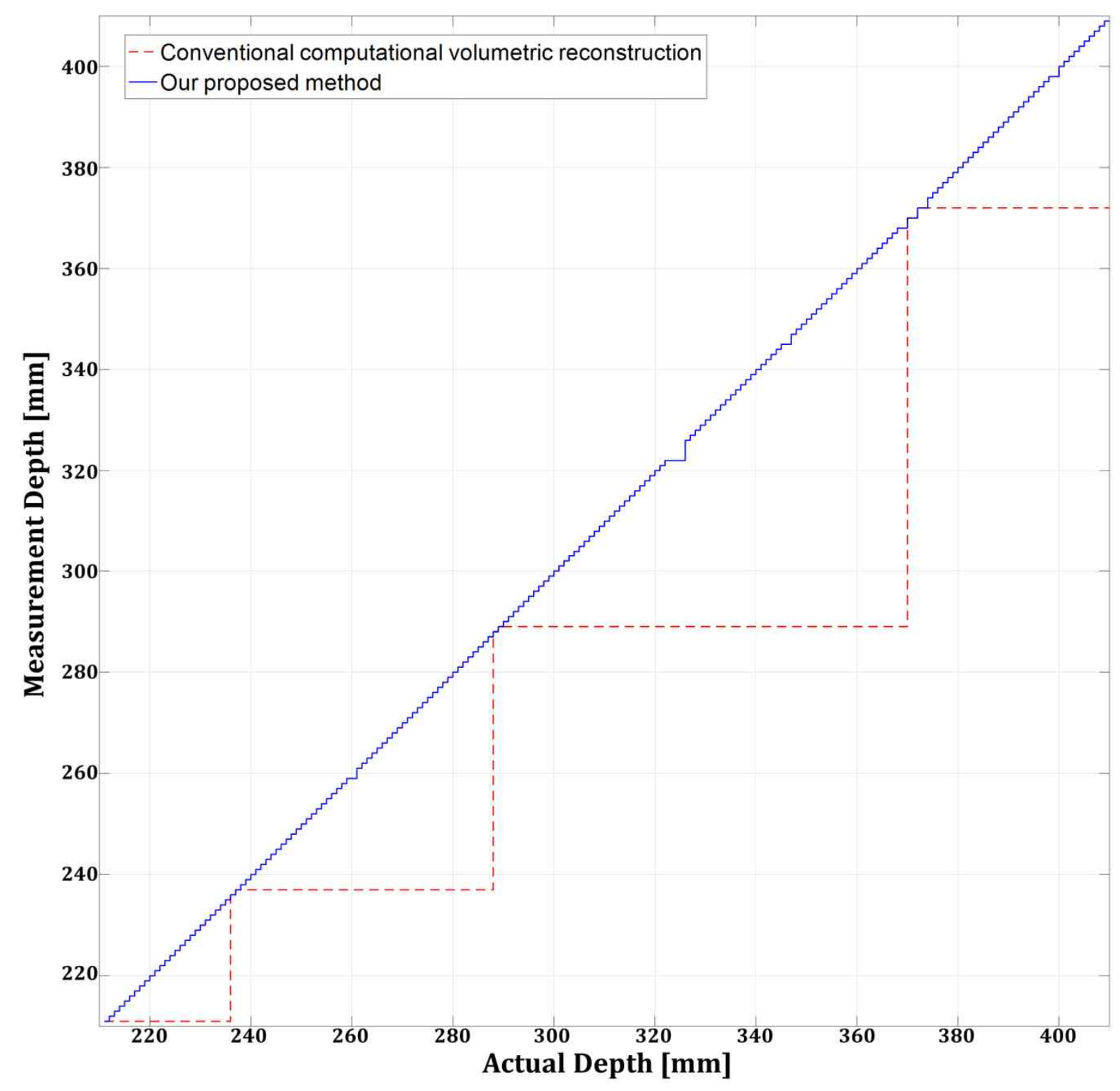

Figure 10. 